\title{
A Clinical Evaluation of Daruharidra (Berberis aristata D.C.) in cases of Giardiasis
}

\author{
Syed Sadiq Abbas*, Agha Parvez Masih, S. Shabihe Raza Baqri, Mohd Yusuf and Kumar Gaurav Bajpai \\ Department of Zoology, Shia P. G. College, Lucknow - 226003, Uttar Pradesh, India; drsadiqabbas@rediffmail.com
}

\begin{abstract}
Daruharidra (Berberis aristata D.C.) has been described in ancient Ayurvedic texts for treatment of Jaundice, Skin diseases and abdominal diseases etc. Hence the present study was carried out in randomly selected cases of stool +ve Giardiasis. The stem and root of Berberis aristata (BA) was administered in capsules after drying in shade and rough grinding. Out of 268 cases with abdominal symptoms and ill health 69 were found +ve for Giardiasis only. These were treated with $1 \mathrm{gm} \mathrm{BD}$ of the Berberis aristata powder for 14 days. Ninety-five percent cases were found free of parasites in stool after treatment. The clinical, Stool microscopic and hematological profiles were studied. The haematological profiles of the patients were improved after 1 month and 1.5 month of treatment. There was significant improvement in clinical profile of patients treated with Berberis aristata. The demographic characteristics of patients were also studied but there was no any correlation between demographic details and infestation of Giardia lamblia.
\end{abstract}

Keywords: Giardiasis, Berberis aristata, Daruharidra, Grahani Roga, Jaundice, Liver disease

\section{Introduction}

Giardiasis is not only a problem of Indian subcontinent but presently it is recognised as a global problem. $12.5 \%$ of world population is suffering from Giardia infection ${ }^{1}$. The manifestation of Giardia is so variable that inspite of large number of studies it is difficult to assess and correlate its clinical effects in man. It starts from asymptomatic condition to severe diarrhoea, malabsorption syndrome and liver necrosis. The effect of Giardia is also well documented for its allergy inducing effects in normal and immunocompromised patients treated with corticosteroids, suffering from renal failure and diabetes mellitus ${ }^{2,3}$. In severely infected individuals symptoms like flatulence, nausea, epigastric discomfort, vomiting belching, anorexia, salivation chronic diarrhoea or constipation have been reported ${ }^{4,5}$. Giardiasis is as old as the Indian civilization as it has been described in ancient Ayurvedic texts (Carak 2700-600 B.C.) as Grahani Roga. A lot of herbals have been prescribed for Grahani Roga in Ayurvedic texts. Although modern drugs viz; Metronidazole and Albendazole are quite effective in Giardiasis ${ }^{4,6}$ but at the same time they produce many side and toxic side effects ${ }^{7}$. The modern drugs also compromise the immune status of the patient while on the other hand the herbals cure the disease and also increase the immunity as well. Thus, Daruharidra was selected for this study to verify the claim of Ayurveda for its use in treatment of Grahani Roga, jaundice and other liver diseases.

\section{Materials and Methods}

A placebo controlled double blind clinical trial was conducted to assess the effectiveness of BA in patients of Giardiasis. The stools of 268 subjects with mild to severe abdominal disturbances were examined for the presence of cysts and trophozoites of Giardia lamblia and other parasites. Among these stools of 49 cases were negative for presence of any parasite, ova and cyst, in 150 cases found multiple infection of Giardia lamblia, Ascaris lumbricoides, Entamoeba histolytica, Enterobius vermicularis, and Escherichia while 69 clearly positive cases were taken for study. They were initially divided into two groups (Group I and Group II).

Group I consisted of 46 adults patients and subdivided into Group Ia and Group Ib. Group Ia consisted of 31 adult patients, 12 males and 19 females with mean age of $45.3 \pm 5.1$ years and $53.1 \pm 4.2$ respectively. All the patients of Group Ia treated with BA in doses of $400 \mathrm{mg}$ TDS orally for a period of 14 days by BA powder filled in gelatin capsules. The clinical profile, stool microscopic and haematological profiles were studied in the patients on pre-treatment day (0 day) and after treatment with BA on days 15, 30 and 45 .

${ }^{*}$ Author for correspondence 
Group Ib consisted of 15 patients 9 males and 6 females with mean age of $39.8 \pm 3.7$ years and $36.2 \pm 4.2$ years respectively. All 15 patients of Group Ib were administered with starch filled capsules in dosage schedule of $400 \mathrm{mg}$ TDS p.o. for a period of 14 days. All the profile including clinical profile, stool microscopic profile and haematological profile was studied for their parameters before treatment day (0 day) and on 15, 30 and 45 days after treatment.

Group II consisted of 23 children and subdivided in Group IIa and IIb. Group Ila consisted of 13 malnourished children, 8 males and 5 females with mean age of $8.9 \pm 2.1$ years and $9.4 \pm$ 1.7 years respectively. All the children of Group IIa presented with malabsorption syndrome with their protruded tummies and anemia with ill health as a prominent feature. The children were physically weak and less active in comparison to other children of their age group. BA powder filled in smaller gelatin capsules and administered in dosage schedule of $200 \mathrm{mg}$ TDS p.o. for a period of 14 days. The clinical profile, stool microscopic profile and haematological profiles were studied on 0 day (before treatment) and on 15, 30 and 45 days after treatment.

Group IIb consisted of 10 Giardia positive children, 5 males and 5 females with mean age of $7.5 \pm 0.8$ and $8.1 \pm 1.4$ years respectively. Group II b was treated with starch filled in smaller identical gelatin capsules in doses of $200 \mathrm{mg}$ TDS p.o. for a period of 14 days. Clinical, stool microscopic profile and haematological profiles were studied on 0 day (before treatment) and on 15, 30 and 45 days after treatment.

Group Ib and IIb later served as Group lc and Ilc and were treated with BA powder in doses of $400 \mathrm{mg}, 200 \mathrm{mg}$ TDS p.o. respectively for a period of 14 days and conducted a cross over study due to ethical reasons. The clinical, stool microscopic profile and haematological profiles were studied on 0 day (before treatment) and on 15, 30 and 45 days after treatment.

The clinical profile was studied for different signs and symptoms profile of patients including diarrhoea, constipation, flatulence, belching, vomiting, anorexia, pyrexia, salivation, abdominal discomfort, nausea, foul smelling stool, abnormal consistency of stool and abnormal frequency of stools etc.

The stool microscopic profile was studied for presence of trophozoites and cysts of Giardia lamblia, mucus, pus cells, R.B.Cs, fat globules, undigested food particles, bacterial outgrowth etc.

The haematological profile was seen for hemoglobin gm\%, Total Leukocyte Count (TLC), Differential Leukocyte Count (DLC) i.e., lymphocytes, polymorphs, monocytes and eosinophil etc. The findings of all the three profiles were observed on 0 day (before treatment) and post treatment days $15,30,45$. Comparisons have been made with 0 day and placebo control groups to see the effectiveness of BA All the data were statistically analysed for its significance. The patients of all the groups were advised to take boiled cooled water during the period of study. The patients positive for multiple infections were treated with modern drugs for their disease.

\section{Results}

The results of Group I are summarized in (Table 1 to 3 ) for Group Ia and (Table 4 to 6 ) for Group Ib and (Table 7 to 9) for Group Ic. Group I consisted of 46 adults and sub divided into Group Ia and Group Ib. The results of Group II are summarized in (Table 10 to 12) for Group IIa and. (Table 13 to 15) for Group IIb and (Table 16 to 18) for Group IIc. Group II consisted of 46 adults and sub divided into Group Ha and Group IIb.

\section{Discussion and Conclusion}

Grahani Roga of Ayurveda appears to be very akin to Giardiasis and Giardia related malabsorption syndrome 8 . It has been treated by various herbal preparations and herbs since long. Giardia, considered to be a harmless parasite in man a few decades back has have been established to cause ill health and malabsorption syndrome in many people particularly in countries like India where reinfections are very common due to poor hygienic conditions and uncured water supply for human consumption. Repeated infections ultimately result in ill health while people with few infections may remain symptomless (asymptomatic) ${ }^{6,9,10}$. The incidence of this disease did not show any correlation with sex, age, weight, food habit, religion and marital status, however, the bad sanitary conditions and poor hygiene show higher incidence.

The results of this study (Tables 1 to 3 and 10 to 12) show significant improvement in clinical profile, stool microscopic profile and blood microscopic profile. A significant increase in hemoglobin contents and decrease in eosinophil count was

Table 1. Group Ia. Effect of BA on Clinical profile (in\%)

\begin{tabular}{|c|c|c|c|c|}
\hline Signs and symptoms & 0 days & 15 day & 30 day & 45 day \\
\hline Diarrhoea & 68 & $10^{*}$ & $7^{\star}$ & $0^{*}$ \\
\hline Abnl. freq. of stool & 87 & $16^{*}$ & $7^{*}$ & $7^{*}$ \\
\hline Foul smelling stool & 100 & $19^{*}$ & $16^{*}$ & $16^{*}$ \\
\hline Abnl. consist of stool & 87 & $13^{*}$ & $7^{\star}$ & $7^{\star}$ \\
\hline Abd. discomfort & 97 & $13^{*}$ & $10^{*}$ & $7^{*}$ \\
\hline Abnl. no. of stool & 100 & $7^{\star}$ & $0^{*}$ & $0^{*}$ \\
\hline Abnormal appetite & 74 & $26^{* *}$ & $10^{*}$ & $7^{*}$ \\
\hline Nausea & 84 & $7^{\star}$ & $10^{*}$ & $7^{*}$ \\
\hline Flatulence & 97 & $19^{*}$ & $10^{*}$ & $10^{*}$ \\
\hline Vomiting & 68 & $10^{*}$ & $0^{*}$ & $0^{*}$ \\
\hline Belching & 74 & $10^{*}$ & $7^{\star}$ & $7^{*}$ \\
\hline Belching & 74 & $10^{*}$ & $7^{*}$ & $7^{\star}$ \\
\hline Anorexia & 74 & $10^{*}$ & $7^{\star}$ & $10^{*}$ \\
\hline Salivation & 65 & $10^{*}$ & $7^{\star}$ & $7^{*}$ \\
\hline Constipation & 29 & $7^{*}$ & $0^{*}$ & $0^{*}$ \\
\hline
\end{tabular}

${ }^{\star} \mathrm{p}<0.01$, ${ }^{* *} \mathrm{p}<0.05$ as compared to 0 day (before treatment) and placebo control. 
observed after 14days treatment with BA. The observations were made on day 15, 30 and 45 in groups Ia and Ila and later Ic and IIc.

The results of placebo groups (Group Ib, Group IIb) shown in Tables 4 to 6 and Tables 13 to 15, There were insignificant changes occurred in all the parameters observed. Only three patients in placebo group $\mathrm{lb}$ and one in placebo group IIb recovered spontaneously as their stools were clear from Giardia cysts and trophozoites. Thus the spontaneous recovery occurs in some cases of Giardiasis.

Group Ib, Group IIb later treated as Group Ic and Group IIc and BA treatment was done in similar way as in Group la and Group Ila as a cross over study for comparison with the same placebo control group. This clinical trial with BA was
Table 2. Group Ia. Effect of BA on Stool microscopic profile (in\%)

\begin{tabular}{|l|l|l|l|l|}
\hline Parasites \& path. Cont. & $\mathbf{0}$ days & $\mathbf{1 5}$ day & $\mathbf{3 0}$ day & $\mathbf{4 5}$ day \\
\hline G. Cysts & 100 & $13^{\star}$ & $10^{\star}$ & $7^{\star}$ \\
\hline G. Tropho. & 23 & $0^{\star}$ & $0^{\star}$ & $0^{\star}$ \\
\hline Mucus & 94 & $19^{\star}$ & $16^{\star}$ & $16^{\star}$ \\
\hline R.B.Cs & 65 & $23^{\star \star}$ & $16^{\star}$ & $13^{\star}$ \\
\hline Pus cells & 68 & $16^{\star \star}$ & $10^{\star}$ & $7^{\star}$ \\
\hline Fat globules & 74 & $19^{\star \star}$ & $7^{\star}$ & $7^{\star}$ \\
\hline U.F.P. & 84 & $10^{\star}$ & $7^{\star}$ & $7^{\star}$ \\
\hline Bacterial outgrowth & 61 & $16^{\star \star}$ & $10^{\star}$ & $10^{\star}$ \\
\hline
\end{tabular}

${ }^{*} \mathrm{p}<0.01$, ${ }^{* *} \mathrm{p}<0.05$ as compared to 0 day (before treatment) and placebo control group.

Table 3. Group Ia. Effect of BA on Haematological profile (in\%)

\begin{tabular}{|l|l|l|l|l|}
\hline Parameters & $\begin{array}{l}\mathbf{0} \text { days } \\
\text { Mean } \pm \text { S.E. }\end{array}$ & $\begin{array}{l}\mathbf{1 5} \text { day } \\
\text { Mean } \pm \text { S.E. }\end{array}$ & $\begin{array}{l}\mathbf{3 0} \text { day } \\
\text { Mean } \pm \text { S.E. }\end{array}$ & $\begin{array}{l}\mathbf{4 5} \text { day } \\
\text { Mean } \pm \text { S.E. }\end{array}$ \\
\hline Haemoglobin & $11.2 \pm 0.3$ & $12.7 \pm 0.3^{* *}$ & $13.5 \pm 0.4^{*}$ & $14.7 \pm 0.4^{*}$ \\
\hline Eosinophil & $9.4 \pm 0.3$ & $6.1 \pm 0.2^{* *}$ & $4.3 \pm 0.2^{*}$ & $3.2 \pm 0.2^{*}$ \\
\hline Lymphocyte & $32.1 \pm 1.4$ & $29.6 \pm 1.3$ & $31.5 \pm 1.3$ & $34.2 \pm 1.3$ \\
\hline Monocyte & $0.5 \pm 0.2$ & $0.8 \pm 0.2$ & $1.0 \pm 0.2$ & $0.5 \pm 0.2$ \\
\hline Polymorph & $62.7 \pm 1.6$ & $60.6 \pm 1.3$ & $63.2 \pm 1.6$ & $62.1 \pm 1.4$ \\
\hline T.L.C. & $8215.0 \pm 81.2$ & $8302.3 \pm 73.6$ & $8134.1 \pm 39.2$ & $8206.2 \pm 28.2$ \\
\hline
\end{tabular}

${ }^{\star} \mathrm{p}<0.01,{ }^{* *} \mathrm{p}<0.05$ as compared to 0 day (before treatment) and placebo control group.

Table 4. Group Ib. Effect of BA on Clinical profile (in\%)

\begin{tabular}{|l|l|l|l|l|}
\hline Signs and symptoms & $\mathbf{0}$ days & $\mathbf{1 5}$ day & $\mathbf{3 0}$ day & $\mathbf{4 5}$ day \\
\hline Diarrhoea & 47 & $47 \#$ & $53 \#$ & $53 \#$ \\
\hline Abnl. freq. of stool & 73 & $67 \#$ & $67 \#$ & $73 \#$ \\
\hline Foul smelling stool & 100 & $93 \#$ & $73 \#$ & $93 \#$ \\
\hline Abnl. consist of stool & 80 & $93 \#$ & $67 \#$ & $73 \#$ \\
\hline Abd. discomfort & 100 & $80 \#$ & $87 \#$ & $93 \#$ \\
\hline Abnl. no. of stool & 93 & $73 \#$ & $87 \#$ & $80 \#$ \\
\hline Abnormal appetite & 87 & $53 \#$ & $73 \#$ & $80 \#$ \\
\hline Nausea & 67 & $67 \#$ & $67 \#$ & $67 \#$ \\
\hline Flatulence & 73 & $53 \#$ & $60 \#$ & $60 \#$ \\
\hline Vomiting & 60 & $67 \#$ & $53 \#$ & $60 \#$ \\
\hline Belching & 73 & $60 \#$ & $60 \#$ & $67 \#$ \\
\hline Anorexia & 53 & $53 \#$ & $60 \#$ \\
\hline Salivation & 60 & $33 \#$ & $53 \#$ & $47 \#$ \\
\hline Constipation & 33 & & $40 \#$ \\
\hline
\end{tabular}

$\# \mathrm{p}=$ NS compared to 0 day (before treatment). 
Table 5. Group Ib. Effect of BA on Stool microscopic profile (in\%)

\begin{tabular}{|l|l|l|l|l|}
\hline Parasites \& path. Cont. & $\mathbf{0}$ days & $\mathbf{1 5}$ day & $\mathbf{3 0}$ day & $\mathbf{4 5}$ day \\
\hline G. Cysts & 100 & $87 \#$ & $80 \#$ & $80 \#$ \\
\hline G. Tropho. & 19 & $20 \#$ & $27 \#$ & $20 \#$ \\
\hline Mucus & 100 & $93 \#$ & $93 \#$ & $87 \#$ \\
\hline R.B.Cs & 60 & $53 \#$ & $60 \#$ & $53 \#$ \\
\hline Pus cells & 73 & $60 \#$ & $73 \#$ & $60 \#$ \\
\hline Fat globules & 67 & $73 \#$ & $73 \#$ \\
\hline U.F.P. & 87 & $53 \#$ & $80 \#$ & $73 \#$ \\
\hline Bacterial outgrowth & 60 & $53 \#$ & $80 \#$ \\
\hline
\end{tabular}

$\# \mathrm{p}=\mathrm{NS}$ compared to 0 day (before treatment).

Table 6. Group Ib. Effect of BA on Haematological profile (in\%)

\begin{tabular}{|l|l|l|l|l|}
\hline Parameters & $\begin{array}{l}\text { O days } \\
\text { Mean } \pm \text { S.E. }\end{array}$ & $\begin{array}{l}\text { 15 day } \\
\text { Mean } \pm \text { S.E. }\end{array}$ & $\begin{array}{l}\text { 30 day } \\
\text { Mean } \pm \text { S.E. }\end{array}$ & $\begin{array}{l}\text { 45 day } \\
\text { Mean } \pm \text { S.E. }\end{array}$ \\
\hline Haemoglobin & $10.8 \pm 0.4$ & $10.5 \pm 0.4 \#$ & $10.3 \pm 0.4 \#$ & $10.6 \pm 0.4 \#$ \\
\hline Eosinophil & $9.5 \pm 0.5$ & $9.2 \pm 0.5 \#$ & $8.7 \pm 0.6 \#$ & $8.4 \pm 0.6 \#$ \\
\hline Lymphocyte & $27.7 \pm 1.4$ & $29.2 \pm 1.2 \#$ & $31.5 \pm 1.3 \#$ & $30.9 \pm 1.2 \#$ \\
\hline Monocyte & $0.4 \pm 0.2$ & $0.8 \pm 0.1 \#$ & $0.5 \pm 0.2 \#$ & $0.6 \pm 0.2 \#$ \\
\hline Polymorph & $63.2 \pm 1.3$ & $60.1 \pm 1.2 \#$ & $61.3 \pm 1.2 \#$ & $60.7 \pm 1.1 \#$ \\
\hline T.L.C. & $8050.2 \pm 50.3$ & $7912.3 \pm 49.5 \#$ & $8130.5 \pm 52.3 \#$ & $8002.3 \pm 48.7 \#$ \\
\hline
\end{tabular}

$\# \mathrm{p}=\mathrm{NS}$ compared to 0 day (before treatment).

Table 7. Group Ic. Effect of BA on Clinical profile (in\%)

\begin{tabular}{|c|c|c|c|c|}
\hline Signs and symptoms & 0 days & 15 day & 30 day & 45 day \\
\hline Diarrhoea & 53 & $0^{*}$ & $0^{*}$ & $0^{*}$ \\
\hline Abnl. freq. of stool & 73 & $13^{*}$ & $6^{*}$ & $0^{*}$ \\
\hline Foul smelling stool & 93 & $20^{*}$ & $13^{*}$ & $13^{*}$ \\
\hline Abnl. consist of stool & 73 & $6^{*}$ & $0^{*}$ & $0^{*}$ \\
\hline Abd. discomfort & 93 & $13^{*}$ & $0^{*}$ & $0^{\star}$ \\
\hline Abnl. no. of stool & 80 & $6^{*}$ & $6^{*}$ & $6^{*}$ \\
\hline Abnormal appetite & 80 & $20^{\star \star}$ & $13^{*}$ & $6^{*}$ \\
\hline Nausea & 67 & $13^{*}$ & $13^{*}$ & $6^{*}$ \\
\hline Flatulence & 60 & $13^{*}$ & $6^{*}$ & $6^{*}$ \\
\hline Vomiting & 60 & $6^{*}$ & $0^{*}$ & $0^{*}$ \\
\hline Belching & 67 & $20^{\star *}$ & $13^{*}$ & $13^{*}$ \\
\hline Anorexia & 60 & $6^{*}$ & $0^{*}$ & $0^{*}$ \\
\hline Salivation & 47 & $6^{*}$ & $13^{*}$ & $6^{*}$ \\
\hline Constipation & 40 & $0^{*}$ & $0^{\star}$ & $0^{\star}$ \\
\hline
\end{tabular}

${ }^{\star} \mathrm{p}<0.01,{ }^{* *} \mathrm{p}<0.05$ as compared to 0 day (before treatment) and placebo control. 
Table 8. Group Ic. Effect of BA on Stool microscopic profile (in\%)

\begin{tabular}{|c|c|c|c|c|}
\hline Parasites \& path. Cont. & 0 days & 15 day & 30 day & 45 day \\
\hline G. Cysts & 80 & $6^{*}$ & $0^{*}$ & $0^{*}$ \\
\hline G. Tropho. & 20 & $0^{*}$ & $0^{*}$ & $0^{*}$ \\
\hline Mucus & 87 & $20^{*}$ & $13^{\star}$ & $0^{*}$ \\
\hline R.B.Cs & 60 & $13^{*}$ & $13^{*}$ & $6^{*}$ \\
\hline Pus cells & 73 & $20^{\star *}$ & $13^{*}$ & $13^{*}$ \\
\hline Fat globules & 73 & $13^{\star}$ & $6^{*}$ & $6^{*}$ \\
\hline U.F.P. & 80 & $20^{*}$ & $20^{*}$ & $13^{*}$ \\
\hline Bacterial outgrowth & 60 & $13^{*}$ & $13^{*}$ & $6^{*}$ \\
\hline
\end{tabular}

${ }^{\star} \mathrm{p}<0.01,{ }^{* *} \mathrm{p}<0.05$ as compared to 0 day (before treatment) and placebo control group.

Table 9. Group Ic. Effect of BA on Haematological profile (in\%)

\begin{tabular}{|l|l|l|l|l|}
\hline Parameters & $\begin{array}{l}\mathbf{0} \text { days } \\
\text { Mean } \pm \text { S.E. }\end{array}$ & $\begin{array}{l}\mathbf{1 5} \text { day } \\
\text { Mean } \pm \text { S.E. }\end{array}$ & $\begin{array}{l}\text { 30 day } \\
\text { Mean } \pm \text { S.E. }\end{array}$ & $\begin{array}{l}\mathbf{4 5} \text { day } \\
\text { Mean } \pm \text { S.E. }\end{array}$ \\
\hline Haemoglobin & $10.6 \pm 0.4$ & $11.8 \pm 0.3^{* *}$ & $12.3 \pm 0.3^{*}$ & $13.6 \pm 0.3^{*}$ \\
\hline Eosinophil & $8.4 \pm 0.6$ & $4.2 \pm 0.3^{* *}$ & $3.1 \pm 0.2^{*}$ & $2.7 \pm 0.2^{*}$ \\
\hline Lymphocyte & $30.9 \pm 1.2$ & $32.2 \pm 1.6$ & $30.7 \pm 1.2$ & $31.2 \pm 1.6$ \\
\hline Monocyte & $0.6 \pm 0.2$ & $0.4 \pm 0.1$ & $0.8 \pm 0.2$ & $0.5 \pm 0.1$ \\
\hline Polymorph & $60.7 \pm 1.1$ & $63.3 \pm 1.7$ & $65.3 \pm 1.5$ & $64.8 \pm 1.4$ \\
\hline T.L.C. & $8002.3 \pm 48.7$ & $8129.2 \pm 37.6$ & $7953.6 \pm 53.2$ & $8263.6 \pm 52.3$ \\
\hline
\end{tabular}

${ }^{\star} \mathrm{p}<0.01,{ }^{* *} \mathrm{p}<0.05$ as compared to 0 day (before treatment) and placebo control group.

Table 10. Group IIa. Effect of BA on Clinical profile (in\%)

\begin{tabular}{|c|c|c|c|c|}
\hline Signs and symptoms & 0 days & 15 day & 30 day & 45 day \\
\hline Diarrhoea & 85 & $0^{*}$ & $0^{*}$ & $0^{*}$ \\
\hline Abnl. freq. of stool & 92 & $15^{*}$ & $0^{*}$ & $0^{*}$ \\
\hline Foul smelling stool & 100 & $31^{\star *}$ & $15^{*}$ & $15^{*}$ \\
\hline Abnl. consist of stool & 100 & $8^{\star}$ & $0^{*}$ & $0^{*}$ \\
\hline Abd. discomfort & 100 & $15^{\star}$ & $0^{*}$ & $0^{*}$ \\
\hline Abnl. no. of stool & 100 & $23^{*}$ & $15^{\star}$ & $15^{\star}$ \\
\hline Abnormal appetite & 85 & $15^{\star}$ & $7^{\star}$ & $7^{\star}$ \\
\hline Nausea & 100 & $15^{\star}$ & $0^{*}$ & $0^{*}$ \\
\hline Flatulence & 92 & $23^{*}$ & $15^{*}$ & $7^{*}$ \\
\hline Vomiting & 62 & $7^{\star}$ & $0^{*}$ & $0^{*}$ \\
\hline Belching & 69 & $15^{\star *}$ & $7^{*}$ & $7^{*}$ \\
\hline Anorexia & 31 & $7^{\star}$ & $0^{*}$ & $0^{*}$ \\
\hline Salivation & 100 & $23^{*}$ & $15^{*}$ & $15^{*}$ \\
\hline Constipation & 15 & $0^{*}$ & $0^{*}$ & $0^{*}$ \\
\hline
\end{tabular}

${ }^{*} \mathrm{p}<0.01,{ }^{* *} \mathrm{p}<0.05$ as compared to 0 day (before treatment) and placebo control. 
Table 11. Group IIa. Effect of BA on Stool microscopic profile (in\%)

\begin{tabular}{|c|c|c|c|c|}
\hline Parasites \& path. Cont. & 0 days & 15 day & 30 day & 45 day \\
\hline G. Cysts & 100 & $7^{\star}$ & $15^{\star}$ & $7^{\star}$ \\
\hline G. Tropho. & 15 & $0^{*}$ & $0^{*}$ & $0^{*}$ \\
\hline Mucus & 92 & $23^{*}$ & $15^{*}$ & $15^{*}$ \\
\hline R.B.Cs & 69 & $15^{*}$ & $7^{\star}$ & $7^{\star}$ \\
\hline Pus cells & 85 & $23^{* *}$ & $15^{\star}$ & $15^{\star}$ \\
\hline Fat globules & 62 & $7^{*}$ & $15^{*}$ & $7^{\star}$ \\
\hline U.F.P. & 92 & $31^{\star \star}$ & $23^{*}$ & $23^{*}$ \\
\hline Bacterial outgrowth & 62 & $15^{\star}$ & $15^{\star}$ & $15^{\star}$ \\
\hline
\end{tabular}

${ }^{\star} \mathrm{p}<0.01,{ }^{* *} \mathrm{p}<0.05$ as compared to 0 day (before treatment) and placebo control.

Table 12. Group IIa. Effect of BA on Haematological profile (in\%)

\begin{tabular}{|l|l|l|l|l|}
\hline Parameters & $\begin{array}{l}\text { 0 days } \\
\text { Mean } \pm \text { S.E. }\end{array}$ & $\begin{array}{l}\text { 15 day } \\
\text { Mean } \pm \text { S.E. }\end{array}$ & $\begin{array}{l}\text { 30 day } \\
\text { Mean } \pm \text { S.E. }\end{array}$ & $\begin{array}{l}\text { day } \\
\text { Mean } \pm \text { S.E. }\end{array}$ \\
\hline Haemoglobin & $7.8 \pm 0.5$ & $9.1 \pm 0.4^{* *}$ & $10.6 \pm 0.3^{*}$ & $12.5 \pm 0.3^{*}$ \\
\hline Eosinophil & $10.2 \pm 0.6$ & $5.1 \pm 0.4^{* *}$ & $3.2 \pm 0.4^{*}$ & $2.0 \pm 0.4^{*}$ \\
\hline Lymphocyte & $28.8 \pm 1.8$ & $31.2 \pm 2.2$ & $29.9 \pm 2.0$ & $34.5 \pm 3.1$ \\
\hline Monocyte & $0.4 \pm 0.4$ & $0.7 \pm 0.3$ & $0.8 \pm 0.3$ & $0.6 \pm 0.3$ \\
\hline Polymorph & $60.8 \pm 3.1$ & $62.3 \pm 3.0$ & $66.4 \pm 1.7$ & $62.8 \pm 3.2$ \\
\hline T.L.C. & $8147.7 \pm 56.2$ & $8423.2 \pm 42.8$ & $8239.5 \pm 54.2$ & $8349.1 \pm 55.2$ \\
\hline
\end{tabular}

${ }^{\star} \mathrm{p}<0.01,{ }^{* *} \mathrm{p}<0.05$ as compared to 0 day (before treatment) and placebo control group.

Table 13. Group IIb. Effect of BA on Clinical profile (in\%)

\begin{tabular}{|l|l|l|l|l|}
\hline Signs and symptoms & $\mathbf{0}$ days & $\mathbf{1 5}$ day & $\mathbf{3 0}$ day & $\mathbf{4 5}$ day \\
\hline Diarrhoea & 60 & $60 \#$ & $70 \#$ \\
\hline Abnl. freq. of stool & 90 & $80 \#$ & $70 \#$ & $80 \#$ \\
\hline Foul smelling stool & 100 & $80 \#$ & $90 \#$ & $90 \#$ \\
\hline Abnl. consist of stool & 90 & $70 \#$ & $80 \#$ & $80 \#$ \\
\hline Abd. discomfort & 100 & $80 \#$ & $70 \#$ & $70 \#$ \\
\hline Abnl. no. of stool & 100 & $70 \#$ & $90 \#$ & $90 \#$ \\
\hline Abnormal appetite & 80 & $60 \#$ & $70 \#$ & $80 \#$ \\
\hline Nausea & 90 & $90 \#$ & $80 \#$ & $70 \#$ \\
\hline Flatulence & 80 & $90 \#$ & $80 \#$ & $80 \#$ \\
\hline Vomiting & 50 & $40 \#$ & $60 \#$ & $60 \#$ \\
\hline Belching & 60 & $50 \#$ & $40 \#$ & $50 \#$ \\
\hline Anorexia & 40 & $50 \#$ & $60 \#$ & $80 \#$ \\
\hline Salivation & 100 & $100 \#$ & $90 \#$ \\
\hline Constipation & 30 & $30 \#$ & $20 \#$ \\
\hline
\end{tabular}

$\# \mathrm{p}=\mathrm{NS}$ as compared to 0 day (before treatment) 
Table 14. Group IIb. Effect of BA on Stool microscopic profile (in\%)

\begin{tabular}{|l|l|l|l|l|}
\hline Parasites \& path. Cont. & $\mathbf{0}$ days & $\mathbf{1 5}$ day & $\mathbf{3 0}$ day & $\mathbf{4 5}$ day \\
\hline G. Cysts & 100 & $90 \#$ & $80 \#$ & $90 \#$ \\
\hline G. Tropho. & 20 & $10 \#$ & $10 \#$ & $20 \#$ \\
\hline Mucus & 90 & $70 \#$ & $70 \#$ & $80 \#$ \\
\hline R.B.Cs & 60 & $50 \#$ & $40 \#$ & $60 \#$ \\
\hline Pus cells & 70 & $80 \#$ & $60 \#$ & $60 \#$ \\
\hline Fat globules & 50 & $40 \#$ & $40 \#$ & $50 \#$ \\
\hline U.F.P. & 60 & $60 \#$ & $50 \#$ & $40 \#$ \\
\hline Bacterial outgrowth & 40 & $40 \#$ & $50 \#$ & $50 \#$ \\
\hline
\end{tabular}

$\# \mathrm{p}=\mathrm{NS}$ as compared to 0 day (before treatment).

Table 15. Group IIb. Effect of BA on Haematological profile (in\%)

\begin{tabular}{|l|l|l|l|l|}
\hline Parameters & $\begin{array}{l}\mathbf{0} \text { days } \\
\text { Mean } \pm \text { S.E. }\end{array}$ & $\begin{array}{l}\mathbf{1 5} \text { day } \\
\text { Mean } \pm \text { S.E. }\end{array}$ & $\begin{array}{l}\text { 30 day } \\
\text { Mean } \pm \text { S.E. }\end{array}$ & $\begin{array}{l}\mathbf{4 5} \text { day } \\
\text { Mean } \pm \text { S.E. }\end{array}$ \\
\hline Haemoglobin & $9.8 \pm 0.2$ & $9.2 \pm 0.3 \#$ & $9.4 \pm 0.3 \#$ & $8.9 \pm 0.3 \#$ \\
\hline Eosinophil & $10.1 \pm 0.6$ & $9.6 \pm 0.8 \#$ & $9.3 \pm 0.7 \#$ & $9.5 \pm 0.7 \#$ \\
\hline Lymphocyte & $28.6 \pm 2.1$ & $20.2 \pm 2.9 \#$ & $30.7 \pm 1.0 \#$ & $28.2 \pm 2.1 \#$ \\
\hline Monocyte & $0.7 \pm 0.3$ & $0.4 \pm 0.7 \#$ & $0.8 \pm 0.3 \#$ & $0.6 \pm 0.6 \#$ \\
\hline Polymorph & $60.7 \pm 3.2$ & $64.3 \pm 2.8 \#$ & $59.8 \pm 4.2 \#$ & $61.3 \pm 3.9 \#$ \\
\hline T.L.C. & $8576.2 \pm 52.3$ & $8729.5 \pm 49.2 \#$ & $8436.0 \pm 56.5 \#$ & $8589.2 \pm 51.6 \#$ \\
\hline
\end{tabular}

$\# \mathrm{p}=\mathrm{NS}$ as compared to 0 day (before treatment).

Table 16. Group IIc. Effect of BA on Clinical profile (in\%)

\begin{tabular}{|c|c|c|c|c|}
\hline Signs and symptoms & 0 days & 15 day & 30 day & 45 day \\
\hline Diarrhoea & 70 & $0^{*}$ & $0^{*}$ & $0^{*}$ \\
\hline Abnl. freq. of stool & 80 & $20^{*}$ & $10^{*}$ & $0^{*}$ \\
\hline Foul smelling stool & 90 & $30^{\star *}$ & $20^{*}$ & $20^{*}$ \\
\hline Abnl. consist of stool & 80 & $20^{*}$ & $10^{*}$ & $10^{\star}$ \\
\hline Abd. discomfort & 70 & $10^{*}$ & $0^{*}$ & $0^{*}$ \\
\hline Abnl. no. of stool & 90 & $20^{*}$ & $20^{*}$ & $10^{*}$ \\
\hline Abnormal appetite & 80 & $10^{*}$ & $10^{*}$ & $0^{*}$ \\
\hline Nausea & 70 & $20^{*}$ & $10^{*}$ & $10^{\star}$ \\
\hline Flatulence & 80 & $30^{\star *}$ & $20^{*}$ & $0^{*}$ \\
\hline Vomiting & 60 & $20^{\star *}$ & $10^{*}$ & $0^{*}$ \\
\hline Belching & 50 & $30^{* *}$ & $20^{* *}$ & $20^{* *}$ \\
\hline Anorexia & 60 & $30^{* *}$ & $20^{\star *}$ & $10^{*}$ \\
\hline Salivation & 90 & $20^{*}$ & $20^{*}$ & $10^{*}$ \\
\hline Constipation & 20 & $0^{*}$ & $0^{*}$ & $0^{*}$ \\
\hline
\end{tabular}

${ }^{*} \mathrm{p}<0.01,{ }^{* *} \mathrm{p}<0.05$ as compared to 0 day (before treatment) and placebo control. 
Table 17. Group IIc. Effect of BA on Stool microscopic profile (in\%)

\begin{tabular}{|c|c|c|c|c|}
\hline Parasites \& path. Cont. & 0 days & 15 day & 30 day & 45 day \\
\hline G. Cysts & 10 & $0^{*}$ & $0^{*}$ & $0^{*}$ \\
\hline G. Tropho. & 90 & $0^{*}$ & $0^{*}$ & $0^{*}$ \\
\hline Mucus & 80 & $30^{* *}$ & $20^{*}$ & $10^{*}$ \\
\hline R.B.Cs & 60 & $20^{\star *}$ & $10^{*}$ & $0^{*}$ \\
\hline Pus cells & 60 & $0^{*}$ & $0^{*}$ & $0^{*}$ \\
\hline Fat globules & 50 & $20^{* *}$ & $0^{*}$ & $0^{*}$ \\
\hline U.F.P. & 40 & $10^{*}$ & $10^{*}$ & $0^{*}$ \\
\hline Bacterial outgrowth & 50 & $20^{*}$ & $10^{*}$ & $10^{*}$ \\
\hline
\end{tabular}

${ }^{\star} \mathrm{p}<0.01,{ }^{* \star} \mathrm{p}<0.05$ as compared to 0 day (before treatment) and placebo control group.

Table 18. Group IIc. Effect of BA on Haematological profile (in\%)

\begin{tabular}{|l|l|l|l|l|}
\hline Parameters & $\begin{array}{l}\text { 0 days } \\
\text { Mean } \pm \text { S.E. }\end{array}$ & $\begin{array}{l}\mathbf{1 5} \text { day } \\
\text { Mean } \pm \text { S.E. }\end{array}$ & $\begin{array}{l}\text { 30 day } \\
\text { Mean } \pm \text { S.E. }\end{array}$ & $\begin{array}{l}\mathbf{4 5} \text { day } \\
\text { Mean } \pm \text { S.E. }\end{array}$ \\
\hline Haemoglobin & $8.9 \pm 0.3$ & $10.3 \pm 0.4^{* *}$ & $11.6 \pm 0.4^{*}$ & $12.5 \pm 0.3^{*}$ \\
\hline Eosinophil & $9.5 \pm 0.7$ & $6.2 \pm 0.3^{* *}$ & $3.4 \pm 0.3^{*}$ & $2.3 \pm 0.3^{*}$ \\
\hline Lymphocyte & $28.2 \pm 2.1$ & $27.8 \pm 2.9$ & $36.5 \pm 1.6$ & $33.8 \pm 1.9$ \\
\hline Monocyte & $0.6 \pm 0.6$ & $0.9 \pm 0.5$ & $0.3 \pm 0.6$ & $0.7 \pm 0.6$ \\
\hline Polymorph & $61.3 \pm 3.9$ & $65.6 \pm 4.5$ & $60.3 \pm 3.5$ & $63.1 \pm 4.2$ \\
\hline T.L.C. & $8589.2 \pm 51.6$ & $8139.5 \pm 45.2$ & $8343.3 \pm 44.3$ & $8563.7 \pm 46.2$ \\
\hline
\end{tabular}

${ }^{*} \mathrm{p}<0.01,{ }^{* *} \mathrm{p}<0.05$ as compared to 0 day (before treatment) and placebo control group.

completed in 69 cases i.e. 21 males, 25 females and 23 children of Giardiasis with significant improvement in all parameters recorded. A comparative study with modern drugs was not thought necessary as the mechanism of action of modern drugs are entirely different. These are usually immunodepressant while herbal drugs act by complex mechanism and are immunomodulatory in nature. All the patients were advised to take boiled water during the period of study and reinfections were not observed. This fact proves this disease to be water borne in the city. Thus the present study indicates large use of $\mathrm{BA}$, an innocuous and cheap herbal preparation in treatment of Giardiasis.

\section{References}

1. Abaza SM, Makhlouf LM, el-Shewy KA, el-Moamly AA. Intestinal opportunistic parasites among different groups of immunocompromised hosts. Journal of the Egyptian Society of Parasitology. 1995; 25(3): 713-27.

2. Chakrapanidutta. CarakSamhita by Agnivesha, with Ayurveda Dipika Commentary edited by Shastri H. $2^{\text {nd }}$ Edn. MotilalBanarasi Das Lahora; 1941.

3. Dalal NJ, et. al. Single dose therapy with metronidazole in Giardiasis. The Indian Practitioner. 1977; 3: 113.

4. Di Prisco MC, Hagel I, Lynch NR, Jimenez JC, Rojas R, Gil M, Mata E. Association between giardiasis and allergy. Annals of
Allergy, Asthma \& Immunology. 1998; 81(3): 261-5. https://doi. org/10.1016/S1081-1206(10)62823-2

5. Goodman LS, Gilman A. Principle of toxocology and treatment of poisoning. Chemical for of drug that produce toxicity, Pharmacological basis of therapeutics. Molinoff PB, Ruddon RW. Mc Grow-Hill companies Luc. U.S.A. $9^{\text {th }}$ Edn; 1996. 65-6.

6. Haralabidis S. Th. Immunodiagnosis of Giadiasis by ELISA and studies on cross-reactivity between the anti Giardialamblia antibodies and some heterologous parasitic antigens and fractions. Annals of Tropical Medicine and Parasitology. 1984; 78(3): 295-300. https://doi.org/10.1080/00034983.1984.11811819. PMid:6486935

7. Kumar R, Nigam P, Sarkari NBS, Gupta AK. Clinical and immunogical aspects of Giardialamblia (A review). The Antiseptic. J Med. Surg. 1989; 86: 440-2.

8. Morecki R, Parker JG. Ultrastructure studies of the human Giardialamblia and adjacent jejunal mucosa in 'a subject with steatorrhoea. Gastroenterology. 1967; 52(2): 151-64. https://doi. org/10.1016/S0016-5085(67)80002-7

9. Om Prakash Introductory remark. The Indian Practitioner. 1970; 2: 121.

10. Singh D. An Anatomical determination of Grahani with special reference to GrahaniRoga. M.D. Thesis, L.U; 1993.

11. Vinayak VK, Khanna R, Kum K. Gut associated immune responses in clinical and experimental giardiasis: an overview. Tropical Gastroenterology. 1990; 11(1): 4-8. 